\title{
EL CÁNCER DE MAMA Y SU RELACIÓN CON LOS FACTORES DE RIESGO MODIFICABLES EN MUJERES DE ARMENIA, QUINDÍO'
}

Jheimy J. García Castañeda. ${ }^{2}$, Bayron Ruiz Hoyos $^{3}$

\section{Resumen}

El cáncer de mama es el cáncer ginecológico más frecuente en mujeres en todo el mundo, con 1,67 millones de nuevos casos reportados. Existen factores de riesgo modificables, como la nuliparidad o la primiparidad luego de los treinta años, no haber lactado, utilizar terapia de reemplazo hormonal, consumo regular de bebidas alcohólicas, obesidad, inactividad física y consumo de tabaco. Objetivo: describir los factores de riesgo modificables en mujeres de Armenia y su relación con cáncer de mama. Metodología: estudio exploratorio con mujeres de una institución prestadora de salud (IPS) privada de Armenia, con cáncer de mama y que consultaron en el segundo semestre de 2013 previo consentimiento informado; se aplicó el instrumento Step V 2.1 previamente validado. Se realizó una prueba piloto, se utilizó la base de datos SPSS 19 y se hizo un análisis univariado y bivariado. Resultados: se incluyeron 77 mujeres con edad promedio 59 años (IC 95\% 56-62), diagnosticadas $26 \%$ en estadio II B y $22,1 \%$ en estadio III B, donde el tratamiento más frecuente fue la mastectomía (67,5\%). De igual forma, se encontraron presentes factores de riesgo como el consumo de tabaco, el alcohol, la obesidad y la inactividad física en esta población. También, se observa relación entre el consumo de grasas saturadas y los estadios avanzados de la enfermedad. Discusión: los resultados son similares a la literatura respecto a los factores de riesgo modificables, a los que se suman los bajos ingresos y el bajo nivel socioeconómico como factores de riesgo de contraer esta patología.

${ }^{1}$ Trabajo Financiado por la Universidad Autónoma de Manizales.

2 Especialista Gerencia de la calidad y Auditoria en Salud. Magíster en Salud Pública. Docente investigadora del Programa de Enfermería, Facultad de Ciencias de la Salud. Grupo de Investigación en Atención Primaria en Salud (GIAPS), Universidad del Quindío. Armenia, Colombia.

${ }^{3}$ Magíster en Educación. Docente del Programa de Medicina. Facultad de Ciencias de la Salud. Universidad del Quindío. Armenia, Colombia. 


\section{BREAST CANCER AND ITS RELATIONSHIP WITH THE MODIFIED RISK FACTORS IN WOMEN OF ARMENIA- QUINDÍO'}

García Castañeda Jheimy J. ${ }^{2}$, Ruiz Hoyos Bayron ${ }^{3}$

\section{Abstract}

Breast cancer is the most common gynecological cancer in women worldwide, with 1.67 million new cases in the world. There are modifiable risk factors, such as nulliparity or primiparity after the age of thirty, not having breast-fed, hormone replacement therapy, regular consumption of alcoholic beverages, obesity, physical inactivity and tobacco consumption. Objective: to describe the modifiable risk factors in women of Armenia and their relationship with breast cancer. Methodology: Exploratory study, with women from a private healthcare institution (IPS) of Armenia, with breast cancer, who consulted in the second half of 2013, with prior informed consent, the previously validated Step V 2.1 instrument was applied. A pilot test was carried out, using the SPSS 19 database, univariate and bivariate analysis. Results: 77 women were included, average age 59 years (95\% CI 56-62), 26\% were diagnosed in stage II B, and $22.1 \%$ in stage III B; the most common treatment was mastectomy (67.5\%). Risk factors such as smoking, alcohol consumption, obesity and physical inactivity are present in this population. There is a relationship between the consumption of saturated fats and the late stages of the disease. Discussion: The results are similar to the literature regarding the modifiable risk factors, besides, a low socioeconomic status, low income are evident risk factors that increase the risk for pathology. 


\section{CÂNCER DE MAMA E SUA RELAÇÃO COM OS FATORES DE RISCO MODIFICÁVEIS EM MULHERES DA ARMÊNIA, QUINDÍO (COLÔMBIA)'}

García Castañeda Jheimy J. ${ }^{2}$, Ruiz Hoyos Bayron ${ }^{3}$

\section{Resumo}

O câncer de mama é o câncer ginecológico mais frequente em mulheres no mundo, com 1,67 milhão de novos casos no mundo. Existem fatores de risco modificáveis, tais como a nuliparidade ou primipridade depois de trinta anos, não ter amamentado, a utilização terapêutica de substituição hormonal, o consumo regular de álcool, obesidade, inatividade física e consumo de tabaco. Objetivo: descrever os fatores de risco modificáveis em mulheres de Armenia e sua relação com o câncer de mama. Metodologia: estudo exploratório com mulheres de uma instituição de saúde privada (IPS) de Armenia, com câncer de mama, que consultaram no segundo semestre de 2013; com consentimento informado prévio, o instrumento Step V $2.1 \mathrm{x}$, previamente validado, foi aplicado. Foi realizado um teste piloto, utilizando a base de dados SPSS 19, análise univariada e bivariada. Resultados: foram incluídas 77 mulheres, com idade média de 59 anos (IC 95\%, 56-62), com diagnóstico de $26 \%$ na fase II B e $22,1 \%$ na fase III B; o tratamento mais frequente foi a mastectomia (67,5\%). Fatores de risco como consumo de tabaco, álcool, obesidade e sedentarismo estão presentes nessa população. Existe uma relação entre o consumo de gorduras saturadas e os estágios avançados da doença. Discussão: os resultados são semelhantes aos da literatura quanto aos fatores de risco modificáveis; além disso, o baixo nível socioeconômico e menores remunerações são fatores de risco evidentes que aumentam o risco nessa patologia. 


\section{Introducción}

El cáncer de mama es el segundo cáncer más común en el mundo y el tipo de cáncer más frecuente entre las mujeres con un estimado de 1,67 millones de nuevos casos diagnosticados en el año 2012, que representan el 25\% de todos los cánceres. También, es el cáncer más común en las mujeres, tanto en las regiones más como en las menos desarrolladas, con una tasa de 883000 casos en las primeras y 794000 en las segundas, según censo del 2012. Este tipo de cáncer es la causa más común de muerte entre las mujeres, en 2012 alcanzó la cifra de 522000 muertes, y el cáncer más frecuentemente diagnosticado en todo el mundo, 140 de 184 países, según datos de la Agencia Internacional para la Investigación en Cáncer IARC (2). Es el primer cáncer en las Américas en cuanto a prevalencia y el segundo en cuanto a mortalidad (2) teniendo en cuenta que en América Latina y el Caribe, tanto el $27 \%$ de los nuevos casos de cáncer como el 15\% de las muertes por esta enfermedad son causadas por el cáncer de mama (2). En Colombia ocupa el segundo lugar en incidencia con 8686 casos registrados en el año 2014 y una tasa de defunciones de $15,3 \%$ entre todos los cánceres femeninos $(3,4)$.

La situación epidemiológica del Quindío, en cuanto a la incidencia durante el período 2001- 2011, registró 255 muertes por cáncer de mama con una tasa de mortalidad de 9,95, superando el índice nacional de 6,58 (5) y figurando entre los 15 departamentos de Colombia con 62 mayor incidencia de cáncer (6). Cada año el número de casos con ese diagnós- tico aumenta. En Quindío residen 1093, el $25,9 \%$ de los casos, desde el 2001. En el 2011 se redujo el números de casos respecto a los años anteriores. El 50\% de las pacientes atendidas son mujeres menores a 60 años (6) y solo para el 2015 se presentaron 120 casos de muerte por cáncer de mama (7).

Por lo anterior, es fundamental trabajar en la prevención de los factores de riesgo modificables con el fin de priorizar las intervenciones que a futuro se puedan realizar en la población objeto, e identificar la magnitud del problema en el colectivo de mujeres. Las acciones de prevención se deben reflejar en los resultados esperados de tal modo que se puedan priorizar aquellas intervenciones en salud pública que limiten el impacto de la enfermedad, retrasen su aparición y controlen sus complicaciones.

De igual manera, además de procurar el diagnóstico oportuno de la enfermedad, se considera de vital importancia determinar los factores de riesgo con el fin de reducir algunos de ellos de forma temprana y efectiva. Algunos de estos factores de riesgo son la obesidad, el aumento de la circunferencia abdominal, el consumo de tabaco, el consumo de alcohol y el consumo de grasas saturadas; que presentan para el Quindío prevalencias similares o superiores a los indicadores nacionales. La prevalencia de obesidad en mujeres en edad fértil es de 15,2 mientras que la medida nacional es del 15,1. La prevalencia de fumadores es del 14,8 cifra que supera el valor nacional que se encuentra en 12,8 ; en cuanto a la prevalencia de consumo de alcohol, esta es del $40,1 \%$ por cada 100000 ha- 
bitantes, mientras que factores protectores, como el consumo de frutas y verduras, se encuentran un $8 \%$ por debajo del nivel nacional con el $40.9 \%$. De acuerdo con lo anterior, se busca determinar la relación existente entre los factores de riesgo mencionados y el cáncer (8).

La obesidad aumenta el riesgo de cáncer de mama en las mujeres posmenopáusicas en un $50 \%$, probablemente por el aumento de las concentraciones séricas de estadio libre (9-11). Del mismo modo, se ha encontrado relación entre el aumento en el radio cintura/cadera y el incremento en el riesgo de padecer cáncer de mama (12), al igual que en las mujeres con IMC > 30 quienes según Lobelo $\mathrm{F}$ et al (10), tienen doble riesgo de padecerlo.

Se demostró que el $14.2 \%$ de las muertes de mujeres por cáncer de mama se asocia a la inactividad física y que existe una reducción del riesgo en un 30\% de quienes realizan actividad física (13-15). El consumo de alcohol es un factor de riesgo relevante en las pacientes que presentan tumores dependientes de estrógenos(10-16). Por otra parte, en relación con el consumo de tabaco se observa que este es un factor de riesgo alto para el desarrollo de cáncer de mama (17-19).

Dada la incidencia de esta enfermedad en la ciudad de Armenia, el acceso de los pacientes a métodos diagnósticos y sus hábitos de vida, se justificó realizar esta investigación en función de conocer y evaluar los factores de riesgo modificables. Considerando que este tipo de factores de riesgo son los que, en conjunto, contribuyen a que el cáncer de mama se manifieste, fue pertinente abordarlos en el presente estudio, máxime cuando en Colombia no se habían realizado investigaciones, hasta el momento, sobre el cáncer de mama y su relación con el sedentarismo, la obesidad y el consumo de alcohol y cigarrillo.

A pesar de que en los diversos estudios se describe la relación entre los factores de riesgo modificables y el cáncer de mama, se observa que en la población de Armenia estos factores de riesgo no han sido tipificados. En este sentido, es donde surge la pregunta que orienta esta investigación: ¿Cuál es la relación entre el cáncer de mama y los factores de riesgo modificables?

\section{Metodología}

La investigación tuvo un enfoque cuantitativo tipo descriptivo de corte transversal, aplicado a una muestra de mujeres con cáncer de mama de una IPS privada de la ciudad de Armenia, Colombia, durante el segundo semestre de 2013. Los datos fueron obtenidos de una encuesta tipo censo donde los criterios de participación que se tuvieron en cuenta fueron los siguientes: mujeres adscritas a IPS privadas, diagnosticadas con cáncer de mama durante el segundo semestre de 2013 y que aceptaron participar de forma voluntaria. Como criterios de exclusión se establecieron mujeres que presentaron alteraciones psiquiátricas, emocionales $\mathrm{o}$ discapacidad mayor que les impidiera participar en el estudio, y mujeres que presentaban otro tipo de cáncer.

Como técnicas e instrumentos de recolección de información se aplicó una en- 
cuesta individual mediante visita domiciliaria y se empleó el instrumento Steps V.2.1 tomado de la página del INC, el cual ya fue validado para Colombia (20). Posteriormente, se realizó un protocolo para medición de peso, talla y medidas antropométricas diseñado por la autora (21).

En cuanto a los aspectos bioéticos, el proyecto fue aprobado por el comité de bioética de la universidad Autónoma de Manizales y el consentimiento informado para participar en el proyecto fue redactado y obtenido de acuerdo con los lineamientos de la resolución 8430 de 1993.

En el Análisis se utilizó la base de datos Spss V19. Se llevó a cabo un análisis Bivariado a partir del cual se identificaron las características sociodemográficas y los porcentajes de los factores de riesgo. Para las variables cualitativas se hizo análisis descriptivo con distribución de frecuencias; las variables cuantitativas se resumieron y posteriormente se calcularon intervalos de confianza.

\section{Resultados}

\author{
Descripción social y demográfica del \\ grupo de participantes
}

Dentro de la población perteneciente a la misma EPS, susceptible de ser encuestada, se calculó un total de 82 mujeres, de las cuales 5 no aceptaron participar en el estudio, por lo que la muestra final fue de 77 . El 59,7\% de la población estudiada residía en la ciudad de Armenia y el resto en las zonas rurales; las edades oscilaban entre los 29 y 86 años con un promedio de 59 años (IC al $95 \%=56$ a 62 ) y un promedio de 3 hijos por paciente. Fueron diagnosticadas en estadio IIB $26 \%$ y en estadio IIIB 22,1\%, el tratamiento más frecuente fue la mastectomía en $67,5 \%$ de la población.

En la Tabla 1 se resumen las características socio-demográficas de la población objeto de estudio:

Tabla 1. Variables sociales y demográficas (N: 77)

\begin{tabular}{|c|c|c|c|c|c|c|}
\hline \multicolumn{2}{|c|}{ Variables } & \multirow{2}{*}{$\frac{N}{51}$} & \multirow{2}{*}{$\begin{array}{r}\text { Porcentajes } \\
66,2 \%\end{array}$} & Variables & \multirow{2}{*}{$\frac{\mathbf{N}}{55}$} & \multirow{2}{*}{$\begin{array}{r}\text { Porcentajes } \\
71,4 \%\end{array}$} \\
\hline & Ama de Casa & & & \multirow[b]{2}{*}{ Régimen } & & \\
\hline \multirow{5}{*}{ Ocupación } & & & & & & \\
\hline & Estudiante & 1 & $1,3 \%$ & Contributivo & 22 & $28,6 \%$ \\
\hline & $\begin{array}{l}\text { Trabajador } \\
\text { independiente }\end{array}$ & 20 & $26 \%$ & Urbano & 46 & $59,7 \%$ \\
\hline & $\begin{array}{l}\text { Empleado } \\
\text { sector Privado }\end{array}$ & 3 & $3,9 \%$ & $\begin{array}{l}\text { Ubicación } \\
\text { Rural }\end{array}$ & 31 & $40,3 \%$ \\
\hline & $\begin{array}{l}\text { Empleado } \\
\text { sector Público }\end{array}$ & 2 & $2,6 \%$ & $<2$ Estrato & 59 & $76,6 \%$ \\
\hline $\begin{array}{l}\text { Estado } \\
\text { civil }\end{array}$ & Viuda & 13 & $16.9 \%$ & $>2$ Estrato & 18 & $23,4 \%$ \\
\hline
\end{tabular}


Continuación Tabla 1. Variables sociales y demográficas (N: 77)

\begin{tabular}{|c|c|c|c|c|c|c|c|}
\hline \multicolumn{2}{|r|}{ Variables } & \multirow{2}{*}{$\frac{\mathbf{N}}{11}$} & \multirow{2}{*}{$\begin{array}{r}\text { Porcentajes } \\
14,3 \%\end{array}$} & \multicolumn{2}{|c|}{ Variables } & \multirow{2}{*}{$\frac{\mathbf{N}}{8}$} & \multirow{2}{*}{$\begin{array}{r}\text { Porcentajes } \\
10,4 \%\end{array}$} \\
\hline \multirow{4}{*}{$\begin{array}{l}\text { Estado } \\
\text { civil }\end{array}$} & Separada & & & & $\begin{array}{l}\text { Sin } \\
\text { escolaridad }\end{array}$ & & \\
\hline & Unión libre & 14 & $18,2 \%$ & & $\begin{array}{l}\text { Primaria } \\
\text { Incompleta }\end{array}$ & 14 & $18,4 \%$ \\
\hline & Casada & 18 & $23,4 \%$ & & $\begin{array}{l}\text { Primaria } \\
\text { Completa }\end{array}$ & 24 & $31,2 \%$ \\
\hline & Soltera & 21 & $27,3 \%$ & $\begin{array}{l}\text { Nivel } \\
\text { educativo }\end{array}$ & $\begin{array}{l}\text { Secundaria } \\
\text { Incompleta }\end{array}$ & 2 & $2,6 \%$ \\
\hline \multirow{3}{*}{ Ingresos } & $\begin{array}{l}<2 \text { salarios } \\
\text { mínimos }\end{array}$ & 63 & $81,8 \%$ & & $\begin{array}{l}\text { Secundaria } \\
\text { Completa }\end{array}$ & 18 & $23,4 \%$ \\
\hline & $\begin{array}{l}\text { >2 salarios } \\
\text { mínimos }\end{array}$ & 14 & $18,2 \%$ & & $\begin{array}{l}\text { Pre } \\
\text { universitario }\end{array}$ & 7 & $9,1 \%$ \\
\hline & & & & & $\begin{array}{l}\text { Universitarios } \\
\text { Completos }\end{array}$ & 4 & $5,2 \%$ \\
\hline
\end{tabular}

Factores de riesgo de cáncer de mama

A. Factores de riesgo no modificables:

a) Antecedentes familiares de cáncer de mama (herencia de cáncer)
El promedio de antecedentes familiares de cáncer de mama, en primer $\mathrm{y}$ segundo grado de consanguinidad, fue de $37,7 \%$.

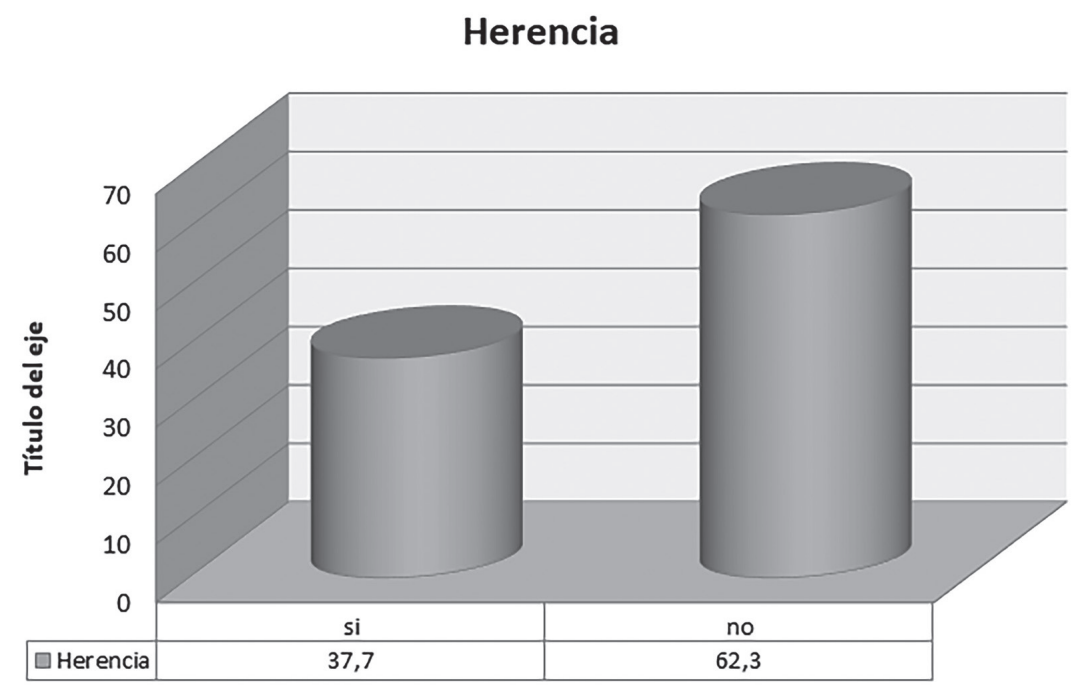

Figura 1. Herencia de cáncer de mama 
B. Factores de riesgo modificables:

El porcentaje de sobrepeso en las pacientes encuestadas fue de $10,4 \%$, la obesidad grado I fue de $6,5 \%$ y la obesidad grado II fue del 1,3\%. En este sentido, se resalta que la relación cintura-cadera anormal se registró en $81,8 \%$ y que las mujeres tuvieron un promedio mayor al 0,9 de relación cintura cadera. (Tabla 2).
En cuanto al promedio de consumo de verduras y frutas se encontró que se realiza en promedio tres días a la semana, lo que demuestra que el consumo de estos alimentos se encuentra por debajo de los límites adecuados que señalan que, para que estos surtan efecto como factores protectores contra el cáncer, su consumo debe realizarse de 5 a 7 días a la semana.

Tabla 2. Factores de riesgo modificables

\begin{tabular}{l|c|c}
\hline \multicolumn{1}{c|}{ Factores modificables } & Frecuencia & Porcentaje \\
\hline Relación cintura-cadera anormal & 63 & $81,8 \%$ \\
Obesidad Abdominal & 54 & $70,1 \%$ \\
Consumo de verduras & 53 & $68,8 \%$ \\
Consumo de frutas & 47 & $61 \%$ \\
Consumo de alcohol & 38 & $49,4 \%$ \\
IMC Alto & 35 & $45,5 \%$ \\
Consumo de cigarrillo & 34 & $44,2 \%$ \\
Inactividad Física & 33 & $42,9 \%$ \\
Consumo de Grasas saturadas & 22 & $28,6 \%$ \\
\hline
\end{tabular}

\section{Análisis bivariado}

Se realizó un análisis bivariado entre los factores de riesgo modificables y la herencia como factor de riesgo no modificable. Con los estadios de la enfermedad y el tratamiento se relacionaron los siguientes factores de riesgo para la producción de cáncer de mama: consumo de alcohol, consumo de tabaco, consumo de grasas saturadas, sobrepeso y obesidad, consumo de frutas y verduras, relación cintura-cadera anormal.
Se encontró una diferencia significativa al relacionar grasas saturadas con los estadios de la enfermedad (Tabla 3 ), fue llamativo encontrar que solo 9 de las 29 pacientes con antecedentes familiares de cáncer de mama se diagnosticaron en estado III. También se realizó un análisis con algunos factores sociodemográficos: estrato socioeconómico, nivel educativo, ubicación de la vivienda y cantidad de ingresos, sin encontrar diferencias significativas. (Tabla 3) 
Tabla 3. Relación de consumo de grasas saturadas con estadios de la enfermedad

\begin{tabular}{|c|c|c|c|c|c|}
\hline & & & $\begin{array}{r}\text { Esta } \\
\text { agrup }\end{array}$ & $\begin{array}{l}\text { dio } \\
\text { ados }\end{array}$ & Total \\
\hline & & & Estadio III y IV & < Estadio III & \\
\hline $\begin{array}{l}\text { Grasas } \\
\text { saturadas }\end{array}$ & $\mathrm{Si}$ & Recuento & 13 & 9 & 22 \\
\hline & & $\begin{array}{l}\% \text { dentro de grasas } \\
\text { saturadas }\end{array}$ & $59,1 \%$ & $40,9 \%$ & $100,0 \%$ \\
\hline & & $\begin{array}{l}\text { \% dentro de Estadio } \\
\text { agrupados }\end{array}$ & $48,1 \%$ & $18,0 \%$ & $28,6 \%$ \\
\hline & No & Recuento & 14 & 41 & 55 \\
\hline & & $\begin{array}{l}\% \text { dentro de grasas } \\
\text { saturadas }\end{array}$ & $25,5 \%$ & $74,5 \%$ & $100,0 \%$ \\
\hline & & $\begin{array}{l}\text { \% dentro de Estadio } \\
\text { agrupados }\end{array}$ & $51,9 \%$ & $82,0 \%$ & $71,4 \%$ \\
\hline Total & & Recuento & 27 & 50 & 77 \\
\hline & & $\begin{array}{l}\text { \% dentro de grasas } \\
\text { saturadas }\end{array}$ & $35,1 \%$ & $64,9 \%$ & $100,0 \%$ \\
\hline & & $\begin{array}{l}\% \text { dentro de Estadio } \\
\text { agrupados }\end{array}$ & $100,0 \%$ & $100,0 \%$ & $100,0 \%$ \\
\hline
\end{tabular}

O.R $=4,230(\mathrm{IC} 95 \%=1,489-12,019)(\mathrm{p}=0,005) \mathrm{X}^{2} 7,808$

Encontramos que existe una relación directamente proporcional entre el consumo de grasas saturadas y los estadios avanzados del cáncer de mama. Se observó que 13 pacientes de la muestra se encuentran en estadio III y IV de cáncer de mama y han sido consumidores regulares de grasas saturadas, mientras que 9 pacientes en estadio $<$ III han consumido grasas saturadas. Por lo cual podemos observar que existe una relación entre el consumo de grasas saturadas y los estadios avanzados de la enfermedad.

\section{Discusión}

Los hallazgos de esta investigación indican que las mujeres que tenían la enfermedad mostraron porcentajes similares de edad a las reportadas en un estudio realizado en Caracas en 2011, en una muestra de 520 pacientes, donde se observó que la población con mayor porcentaje de cáncer se encuentra en edades entre 40 y 64 años con un 74 \% (22). Además, al realizar comparaciones con estudios nacionales se encontró que los 
grupos etarios con mayor riesgo son las mujeres mayores de 40 años (22).

En un estudio realizado en Norteamérica, el occidente de Europa, Asia y Sur América, se mostró la contribución de diversos factores de riesgo modificables, con excepción de factores reproductivos, a la carga global de cáncer de mama (23). Los autores concluyen que el $21 \%$ de todas las muertes registradas por cáncer de mama son atribuibles al consumo de alcohol, el sobrepeso, la obesidad y la falta de actividad física (23).

En el presente estudio se observa que existe un gran porcentaje de mujeres que presentan obesidad, la relación de cintura cadera se encuentra elevada y compromete al $81,8 \%$ del total de la muestra. Adicionalmente, la obesidad abdominal se encuentra presente en el 70,5\% de la población, lo que coincide con un estudio en que las mujeres posmenopáusicas demuestran tener una asociación entre obesidad y cáncer de mama, sobre todo de la obesidad abdominal $(25,25)$. Frente al sobrepeso y la obesidad, en el presente estudio se encontró un porcentaje de sobrepeso del $37,7 \%$, cifra similar a un estudio realizado en el año 2010, en el que se observó que un $34,3 \%$ de la población presenta sobrepeso (26). En cuanto a la obesidad, se encontró que está presente en el 7,8\% del total de la muestra, con un $6,5 \%$ en grado I y el 1,3\% restante en grado II. Lo anterior, evidencia ausencia de obesidad en un alto porcentaje del total de la muestra, sin descartar la existencia del sobre peso como factor de riesgo predominante.

El consumo de alcohol en este estudio alcanzó un porcentaje del 49,2\%. Diver- sos estudios epidemiológicos muestran la relación del consumo de alcohol con el cáncer de mama para tumores receptores de estrógenos $(26,27)$. En países como México la ingesta de alcohol es un factor dietario que ha tenido un papel más relevante en la incidencia de cáncer de mama.

En un meta-análisis de más de 40 informes y 41477 casos incidentes de cáncer de mama, se comparó a las mujeres no bebedoras con aquellas que consumían una bebida con $12 \mathrm{~g}$ de contenido de alcohol. Se encontró que en un $10 \%$ de la muestra [95\% CI $=6 \%-14 \%]$ aumentó el riesgo de cáncer de mama; en dicho estudio se observó que existe relación entre el cáncer de mama y el papel de la adiposidad, la actividad física y la dieta sobre el riesgo de cáncer de mama en mujeres mexicanas (27). Según las estadísticas colombianas, el promedio de inicio de consumo de alcohol y de tabaco en nuestra población se encuentra alrededor de los 13 años. El aumento del IMC y el porcentaje consumo de grasas saturadas son datos que reflejan la realidad de estos factores de riesgo en nuestra población (24).

El consumo de cigarrillo en este estudio se encontró en un 44,2\% de la población, cifra mayor que en un estudio de casos y controles realizado con 130 y 150 pacientes en México en el 2012, donde el antecedente de tabaquismo fue positivo en $29,6 \%$ (25). En un metanálisis sobre cáncer de mama se revisaron 19 estudios, que concluyeron que el cigarrillo es un factor de riesgo tanto para las fumadoras activas como para las fumadoras pasivas (26-28). 
Aunque el consumo de grasas saturadas no fue muy elevado en este estudio $28,6 \%$, es importante resaltar que en otros se ha visto relación entre el consumo de grasas saturadas y el cáncer de mama (28-30). En México, dos estudios de casos y controles demostraron una asociación entre grasa saturada y la presencia de cáncer, donde además se evidenció, una asociación inversa para grasas poli insaturadas entre las mujeres posmenopáusicas al comparar los cuartiles extremos de la ingesta $(30,31)$.

La relación entre el consumo de grasa y el riesgo de padecer cáncer de mama es mixta, de acuerdo con estudios internacionales de correlación en países con una alta incidencia de cáncer de mama, como los EE.UU., donde se observa que la grasa contribuye alrededor del 34\% de las calorías totales; mientras que en países con una baja incidencia de cáncer de mama, como China, la grasa aporta muchas menos calorías, entre 15\%y $20 \%$ de las calorías totales (31).

La falta de actividad física, una dieta alta en hidratos de carbono y una alta carga glicémica, se han relacionado con un aumento en el riesgo de cáncer de mama en mujeres Mexicanas (31).

\section{Conclusiones}

El estudio reportó que las mujeres con sobrepeso o con algún grado de obesidad tienen más riesgos de padecer cáncer de mama. Por otra parte, el consumo de alcohol y tabaco se reportan como factores de riesgo considerables que generan cáncer de mama debido al tipo de componentes químicos que las componen.
El estudio es el primer reporte de factores de riesgo que se realiza en la población de mujeres con cáncer de mama en Armenia, Quindío. Se observó que existe una relación entre el consumo de grasas saturadas y los estadios avanzados de la enfermedad.

Por otra parte, no se encontró una relación directa entre el consumo de tabaco, el alcohol, la circunferencia de cintura cadera y los estadios avanzados del cáncer de mama.

\section{Recomendaciones}

Esta investigación puede servir como línea base para la comparación futura de resultados, modo tal que se justificaría diseñar, implementar y ejecutar programas, de vigilancia, monitoreo $y$ educación relacionados con los factores de riesgo de cáncer de mama, en el marco del plan decenal de salud, para la población de mujeres residentes en Armenia.

No se conocen estudios sobre casos y controles en cáncer de mama en la población de Armenia, por lo que podría ser interesante profundizar en los conocimientos de los factores de riesgo de cáncer de mama encontrados en nuestro estudio.

Se observa que muchas de las mujeres no poseen apoyo en cuento al manejo de la enfermedad, por lo cual se propone que tanto las universidades como los servicios de salud de la zona se encarguen de promover la creación de programas orientados a apoyar a las pacientes en el conocimiento y manejo de su enferme- 
dad, así como de la orientación en trámites en salud y apoyo terapéutico.

Se recomienda realizar en las comunidades trabajos de promoción y prevención de la enfermedad, tal como lo plantea nuestra legislación en salud, con el fin de contribuir con la búsqueda activa de las usuarias que podrían presentar signos y síntomas de la enfermedad de forma temprana y que podrían ser sub-diagnosticadas.

\section{Referencias}

1. Wiesner C. Determinantes psicológicos, sociales y clínicos del cáncer de mama Detección Temprana en Bogotá, Colombia [Internet]. Rev. Col cancerología 2007; 11(1):13-22. [Consulta 9 de febrero 2015]..http://www.scielo.org.mx/scielo. php?script=sci_nlinks\&ref $=7055560 \&$ pi$d=S 0036-36342009000800016000$

2. The World Bank Group 2014 Indicators. Breast Cancer Estimated Incidence, Mortality and Prevalence Worldwide in 2012. [Internet]. [Consultado 9 de febrero 2015]. Disponible en http://globocan.iarc. $\mathrm{fr} / \mathrm{old} /$ FactSheets/cancers/breast-new. asp

3. Agencia Internacional para la Investigación sobre el Cáncer. Detección del cáncer de mama. Manuales IARC de la Prevención del Cáncer, 2002;7.

4 . Ministerio de la Protección Social; Instituto Nacional de Cancerología. [Internet]. [Consultado 13 febrero 2018]. Disponible en: http://www.cancer.gov.co/ documentos/Incidencia/Tabla\%2048.pdf 2008; 19.

5. López C., Londoño N., Ospina M., et al. Análisis de la situación de salud del departamento del Quindío en el modelo de los determinantes sociales de la salud. Gobernación del Quindío. 2014. Disponible en https://quindio.gov.co/home/ ASIS_QUINDIO_2016_ACTUALIZADO_ FINAL_ac_1.compressed.pdf
6. Martínez J., Moreno G., Londoño P. Tendencia en el reporte de casos de cáncer en Oncólogos del Occidente, Pereira, Colombia. Revista Médica de Risaralda [Internet] [Consultado 9 de febrero 2015]. Disponible en: http://revistas.utp.edu.co/ index.php/revistamedica/article/viewFile/7867/4805. 2012; (18):116-21.

7. Rodríguez J. Descripción de la mortalidad por departamentos Colombia año 2000, [Internet], Bogotá, CENDEX [consultado 5 de septiembre 2013]. Disponible en http://www.javeriana.edu.co/cendex/ pdf/DT\%20016-05.pdf.

8. Tamayo F., Gómez A., Plata M., et al. Encuesta Nacional de Salud y Demografía. Bogotá (Colombia) [Internet]. [Consultado 28 de febrero 2016]. Disponible en: https://dhsprogram.com/pubs/pdf/FR246/FR246.pdf

9. Bergoc A., Quartucci R., Outomuro A. et al. Factores de riesgo convencional y emergente en cáncer de mama: un estudio en pacientes posmenopáusicas [Internet]. Rev chil obstet ginecol. 2009; 74(3):135-42. Disponible en: https://scielo.conicyt.cl/scielo.php?script=sci_arttext\&pid $=S 0717-75262009000300002$

10. Lobelo F., Pate R., Parra D. Carga de mortalidad asociada a la inactividad física en Bogotá [Internet]. Rev. Salud Pública. 2006; 8 (2):28-41 Disponible en: http://www.redalyc.org/ pdf/422/42209903.pdf 
11. Tjonneland A., Christensen J., Olsen A., et al. Alcohol intake and breast cancer risk: the European Prospective Investigation into Cancer and Nutrition (EPIC). Cancer Causes Control 2007; (18):361-73. Citado por: de Romieu I, Lajous M.The role of obesity, physical activity and dietary factors on the risk for breast cancer: Mexican experience.Salud Pública Mex 2009; 51 volumen (2):172-180.

12. Aguilar C., Neri M., Padilla L., et al. Factores de riesgo como pronóstico de padecer cáncer de mama en un estado de México [Internet]. 2012, [cited May 13, 2014]; Disponible en: http://dx.doi. org/10.3305/nh.2012.27.5.5997 .

13. Heredia M., Bautista L., Pérez R. Factores de riesgo para cáncer de mama. Revisión de la literatura: Rol potencial de Enfermería. Revista de la Facultad de Medicina de la UNAM [Internet]. 2007, [cited May 13, 2014]; 50 (3): 110-14. Disponible en: http://www.revistas.unam.mx/index. php/reu/article/view/29937.

14. Coronado G., Beasley J., Livaudais $\mathrm{J}$. Alcohol consumption and the risk of breast cancer. Salud Pública de México [Internet]. [cited May 13, 2014]; 53(5): 440-47. Disponible en: https://www.ncbi. nlm.nih.gov/pubmed/22218798

15 República de Colombia. Ley 1384 del 2010. Ley Sandra Ceballos, por la cual se establecen las acciones para la atención integral del cáncer en Colombia [Internet]. [Consultado 9 de febrero 2015] Disponible en: http://190.147.213.68:8080/homepage/alegis_inter/leyes_y_decretos/2010/ ley_1384_de_2010.pdf

16. República de Colombia. Ministerio de la protección social. Plan Decenal para el Control del Cáncer en Colombia, 20122021. Ministerio de Salud y Protección Social - Instituto Nacional de Cancerología [Internet].[Consultado 11 de octubre 2013]. Disponible en http://www.cancer. gov.co/documentos/Plandecenalparaelcontroldelcancer/PlanDecenal_ControlCancer_2012-2021.pdf.
17. Departamento de salud y servicios humanos de EE. UU. Institutos Nacionales de la Salud. Lo que usted necesita saber del cáncer de seno. [Internet]. [Consultado 15 de septiembre del 2013] http://www. cancer.gov/espanol/tipos/necesita-saber/ seno.pdf.

18. Sardiñas R. Autoexamen de Mama: Un importante instrumento de Prevención del Cáncer de Mama en Atención Primaria de Salud. Policlínico Universitario Rampa. 2007:1-10.

19. Instituto Nacional de Cancerología. Recomendaciones para la tamización y la detección temprana del cáncer de mama en Colombia. Guía clínica [internet]; 2006 [Consultado el 19 de mayo 2014]. Disponible en: http://www.cancer.gov.co/ documentos/RecomendacionesyGuias/ GuiaN1.pdf

20. Smith R. American Cancer Society Guidelines for Breast Cancer Screening: Update 2003. CA Cancer J Clin 2003:54:141-169.

21. República de Colombia Ministerio de Protección Social., Instituto Nacional de Cancerología: Recomendaciones para la detección temprana del cáncer de mama [internet] Bogotá: Inc. c2006 [consultado el 9 de febrero del 2015];1-41. Disponible en:http://www.fundayama.org.co/documentacion/GUIA\%20DETECCION\%20 TEMPRANA\%20MAMA\%20INC.pdf

22. Organización Mundial de la Salud. Manual del método STEP wise para la vigilancia de enfermedades crónicas. Parte 3: Guías para la formación e instrucciones prácticas [Internet]. [Consultado enero 26 de 2010]. Disponible en: http://www.who.int/chp/steps/manual/es/ index1.html

23. Fajardo M. Manual de antropometría [internet]. [consultado 15 junio del 2013] disponible en:http://www.inder.cu/indernet/Provincias/hlg/documetos/textos/MEDICINA\%20DEPORTIVA/MEDICINA\%20 
24. Organización panamericana de Salud. Indicadores básicos de salud situación de salud en Colombia [Internet]. 2011 [Consultado el 2 de Julio 2014]. Disponible en: https://hiss.paho.org/ pahosys/grp.php

25 Curado M., Edwards B., Shin H. Cancer Incidence in five continents. Lyon (France): International Agency for Research on Cancer [Internet]. 2007 [Consultado el 2529 septiembre 2008]. Disponible en: https://www.iarc.fr/en/publications/pdfs-online/epi/sp160/Cl5vol9.pdf

26.Frenk J., González E., Gomez O., et al. Health system reform in Mexico 1. Comprehensive reform to improve health system performance in Mexico Lancet 2006; 368:1524-1534

27. Boyd N., Stone J., Vogt K., et al. Dietary fat and breast cancer risk revisited: a meta-analysis of the published literature. Br J Cáncer 2003;89 (9):1672-85

28. Aguilar M., Neri M., Padilla C, et al. Factores de riesgo como pronóstico de padecer cáncer de mama en un estado de México. Nutrición Hospitalaria [Internet].
2012 sep [Citado febrero 20 2015]; 27(5): 1631-1636.

29. Crovetto M, Uauy R. Reflexiones sobre las recomendaciones en salud pública para la prevención del cáncer dadas por el fondo mundial para la investigación sobre cáncer (FMIC) y la situación de Chile. Archivos Latinoamericanos de Nutrición [Internet]. 2014 junio; [Citado febrero 20 de 2015]; 64(2): 83-90. Disponible en: http://www.scielo. org.ve/scielo.php?script=sci_arttext\&pi$d=$ S0004-06222014000200002

30. Romieu I., Lajous M. The role of obesity, physical activity and dietary factors on the risk for breast cancer: Mexican experience [Internet]. Salud Pública Mex 2009;51(2):172-80. [Citado 15 febrero]. Disponible en: https://pdfs.semanticscholar.org/d457/ee1f81f83c67898e9e292b098aa535f54ea7.pdf

31. Coronado G., Beasley J., Livaudais D. Consumo de alcohol y riesgo de cáncer de mama. Rev. Salud Pública Mex. 2011;53:440-447. http://www.medigraphic.com/pdfs/salpubmex/sal-2011/ sal115j.pdf 